\title{
VORSCHAU 10
}

\section{ENTWICKLUNG}

Innovatives Funktionsmanagement
per Touchpad

Datensicherheit und nachladbare HMls

Einzelradlenkung des Forschungsfahrzeugs SpeedE

Effizienz bei Nutzfahrzeugen mit Energierückgewinnung und Aerodynamik

\section{SPECIAL PRODUKTION}

Ergonomie in der Produktion

Kugelzapfen aus nichtrostendem Stahl im Pkw-Fahrwerk

Auswirkungen des MultimaterialLeichtbaus auf die Fügetechnik

Werkreportage Manufaktur für den Porsche 918 Spyder

\section{FORSCHUNG}

Einfluss von Überlasten auf die Lebensdauer von Punktschweißverbindungen

Energieeffizienter Antriebsstrang durch Reibungsminderung

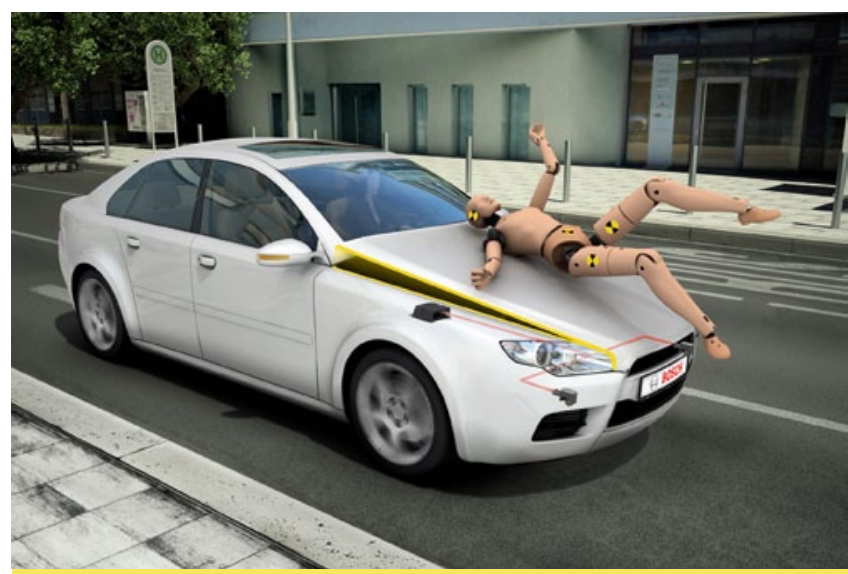

Komfortfunktionen werden mit Aufgaben der aktiven und passiven Sicherheit verzahnt. Treiber sind die Technikführerschaft unter den OEMs und die Gesetzgebung à la Euro NCAP. ABS und ESP waren gestern, heute sind Assistenzsysteme gefragt, um das Fahren einfacher zu machen und die Unfallzahlen zu reduzieren.

Mit der Entwicklung eines Beltbags für den Fahrzeugfond schlägt Daimler ein neues Kapitel für die Fahrzeugsicherheit auf. Der speziell gestaltete Sicherheitsgurt, dessen Gurtband sich mit Gas befüllen lässt, kann das Risiko von Rippenverletzungen deutlich reduzieren. Die so erreichte Reduzierung der Brustkorbbelastung verringert die Verletzungsschwere.

Der Schutz schwacher Verkehrsteilnehmer wie Fußgänger und Fahrradfahrer erfordert weitere Innovationen. Gemeinsam mit Autoliv entwickelte der niederländische Entwicklungsdienstleister TNO dazu einen neuartigen Windschutzscheibenairbag sowie eine Erweiterung der Notbremsfunktion.

Im Interview mit der ATZ beantwortet Gerhard Steiger, Vorsitzender des Geschäftsbereichs Chassis Systems Control bei Bosch, Fragen zur aktiven und passiven Sicherheit, zu Assistenzsystemen und deren Verknüpfung sowie zum automatisierten Fahren. 